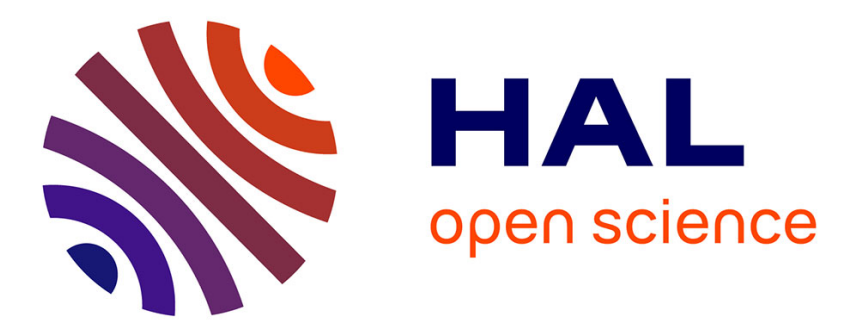

\title{
Deformation modes and size effect in near- $\gamma$ TiAl alloys
} Jean-Philippe Monchoux, Jiangshan Luo, Thomas Voisin, Alain Couret

\section{To cite this version:}

Jean-Philippe Monchoux, Jiangshan Luo, Thomas Voisin, Alain Couret. Deformation modes and size effect in near- $\gamma$ TiAl alloys. Materials Science and Engineering: A, 2017, 679, pp.123 - 132 . 10.1016/j.msea.2016.09.092 . hal-01907480

\section{HAL Id: hal-01907480 \\ https://hal.science/hal-01907480}

Submitted on 29 Oct 2018

HAL is a multi-disciplinary open access archive for the deposit and dissemination of scientific research documents, whether they are published or not. The documents may come from teaching and research institutions in France or abroad, or from public or private research centers.
L'archive ouverte pluridisciplinaire HAL, est destinée au dépôt et à la diffusion de documents scientifiques de niveau recherche, publiés ou non, émanant des établissements d'enseignement et de recherche français ou étrangers, des laboratoires publics ou privés. 


\title{
Deformation modes and size effect in near- $\gamma$ TiAl alloys
}

Jean-Philippe Monchoux ${ }^{(1)}$, Jiangshan Luo ${ }^{(1,2)}$, Thomas Voisin ${ }^{(1)}$, Alain Couret ${ }^{(1)}$

(1) CEMES, CNRS UPR 8011 et Université de Toulouse, 29 rue Jeanne Marvig, 31055 Toulouse, France

(2) Permanent address: Research Center of Laser Fusion, CAEP, P.O. box 919-987, 621900, Mianyang, China

* Corresponding author : monchoux@cemes.fr

\begin{abstract}
This paper presents a study of a size effect in TiAl alloys. Spark Plasma Sintering is used to sinter several alloys with a near- $\gamma$ microstructure. Both the effect of boron as an inhibitor of grain growth and the rapidity of this powder metallurgy process are used to control the grain size. Following a comparison between EBSD-SEM (Electron Backscatter Diffraction by Scanning Electron Microscopy) and TEM (Transmission Electron Microscopy), the grain size is measured by TEM. Tensile tests are performed at room temperature to measure the yield stress. The deformation microstructure is studied by TEM. In a delimited area, activated deformation systems are identified in every grain. Then, for every system, the Schmid's factors as well as the defect nucleation sites are determined. A size effect leading to a high Hall-Petch constant of $\mathrm{k}=0.98 \mathrm{MPa} \mathrm{m}^{1 / 2}$ is measured. The deformation is found to be due to ordinary dislocations and twinning, which are mainly satisfying Schmid's law. Ordinary dislocations are more frequently observed than twins. Twins are found to form pile-ups and to cross the boundaries under the effect of stress concentration. These results are discussed and interpreted on the basis of the pile-up model applied to the Shockley dislocations involved in twinning.
\end{abstract}

\section{Keywords}

Size effects; Titanium aluminides; Mechanical properties, Twinning; Dislocations, 


\section{Introduction}

Size effects in metals and alloys, i.e. relationships between grain size and a material's strength, have been the subject of many studies with nanostructured materials. However, size effects also play an important role in structural materials, where the characteristic dimensions are greater than one micron. Mastering grain size appears thus as a way to enhance the strength of the alloys. This is particularly the case of TiAl alloys, which are mainly developed for high temperature applications in the aeronautic or automotive industries. As reviewed by Appel et al. [1], the strengthening of several TiAl alloys, with various microstructures and grain sizes, can be described by Hall-Petch plots. At room temperature, for a given composition, the yield stress was found to be driven by either the grain size or the lamellar spacing, in the case of equiaxed [2] or lamellar [3] microstructures, respectively. Likewise, creep tests, reproducing the thermal/mechanical solicitation imposed on the material during its lifecycle, highlighted that the creep rate of a fully lamellar Ti-47 Al alloy was a function of the lamellae width [4].

The present work aimed to investigate a size effect in a near- $\gamma \operatorname{TiAl}_{47} \mathrm{Cr}_{2} \mathrm{Nb}_{2}$ alloy with a simple microstructure. The microstructure was indeed largely dominated by $\gamma$ grains, as confirmed by volume fraction measurements (section II) revealing an $\alpha_{2}$ phase content as low as $3.5 \%$. With such microstructures, and given that the $\alpha_{2}$ phase does not usually deform easily, the plastic deformation always occurs in the $\gamma$ phase. Samples with various $\gamma$ grain sizes were produced by Spark Plasma Sintering (SPS) [5], combining the effect of boron addition and the rapidity of this process to control the grain growth [6].

\section{Grain size measurement in $\gamma$ TiAl alloys}

In order to quantify a size effect, it is necessary to measure the grain size. The $\gamma$ phase in TiAl alloy has a quadratic ordered $\mathrm{L} 1_{0}$ structure, with six possible orientation variants that can be separated in a same grain by either ordered domain boundaries or twin boundaries [7]. The former take a curved shape whereas the latter appear to be rectilinear, as the stacking fault associated with the twin interface lies in a (111) plane. To detect all the grain boundaries and calculate the grain size, conventional electron diffraction methods are generally used. The difficulty is to separate the six orientation variants from each other, which differ by stacking sequence and/or long-range order. EBSD-SEM based on the analysis of Kikuchi patterns is currently the most popular technique. To validate the EBSD measurements, the same zone was scrutinized by Transmission Electron Microscopy (TEM) and by EBSD (Fig. 1). Figure 
1a shows this area imaged by TEM. This view is a montage of more than one hundred micrographs, which were taken without tilting the TEM holder more than 10 degrees and without removing the specimen from the holder. Sometimes, complementary micrographs were taken under different tilt conditions in order to achieve a better delimitation of the grains. This zone contains $119 \gamma$ grains and $16 \alpha_{2}$ grains, colored in Fig. 1b. The $\alpha_{2}$ grains (in black) represent $3.5 \%$ of the volume fraction. Note that the color code does not reflect the grains' orientations, which were not determined by TEM. The $\gamma$ grain size spreads between $0.5 \mu \mathrm{m}$ and $6 \mu \mathrm{m}$ with an average of $1.7 \mu \mathrm{m}$. Fig. 1c shows the EBSD map of the same area. Grains colored in blue and green in figure $1 \mathrm{~b}$ are those which were also recognized by EBSD. On the other hand, those in red, pink and yellow were not recognized by EBSD. In fact, grains which were not detected by EBSD were found to be of three types, as underlined by the enlargements (Fig. 1d, e \& f). First, the unseparated grains were ordered domain related as illustrated by the dark field micrographs taken with superlattice reflection operating (Fig. 1d). Second, many rectilinear growth-twin boundaries were not detected by EBSD (Fig. 1e). The reason for this error could be the confusion between twins and pseudo symmetries by the software. Third, some relatively small grains were not detected (Fig. 1f). Note that this mistake did not result from an insufficient resolution $(0.2 \mu \mathrm{m})$ since it is largely lower that the size of these grains $(\sim 1 \mu \mathrm{m})$. Due to these experimental limitations to measure grain size by EBSD-SEM, all the measurements performed during this work were made on TEM pictures, even though it is detrimental to the total number of analyzed grains. Nevertheless, the sizes of around 120 grains belonging to a unique zone were measured for each specimen. The grain size was considered to be the diameter of a circle having the same surface area as the measured grain.

\section{Microstructures and tensile tests}

Two batches of powder with the so-called GE compositions were used: a boron-free powder $\left(\mathrm{TiAl}_{47} \mathrm{Cr}_{2} \mathrm{Nb}_{2}\right.$ - labeled GE) and a boron-containing powder $\left(\mathrm{TiAl}_{47} \mathrm{Cr}_{2} \mathrm{Nb}_{2} \mathrm{~B}_{0.6}-\right.$ labeled GEB) [6]. Four specimens with a near- $\gamma$ microstructure (predominantly formed by $\gamma$ grains) were densified (Tab.1). Fig. 2 shows their microstructure. Specimen temperatures $\mathrm{T}_{\mathrm{s}}$ were determined from the temperature measured by an external pyrometer using the correction calibrated by crossing the $\alpha$ transus [5]. In both alloys, the small $\alpha_{2}$ grains (bright) are situated at the periphery of the $\gamma$ grains (dark). For the GEB alloy, at $\mathrm{T}_{\mathrm{s}}=1200^{\circ} \mathrm{C}$, the periphery of the former powder particles is decorated by $\alpha_{2}$ grains (Fig.2 a). These microstructures displayed some heterogeneities in the distribution of $\alpha_{2}$ grains, which result 
from the dendritic structure of the atomized powder particles. These micrographs revealed a growth of both $\gamma$ and $\alpha_{2}$ grains at increasing temperature.

Fig. 2e displays the tensile curves at room temperature of these four specimens. The corresponding tensile tests were performed at a constant strain rate of $10^{-4} \mathrm{~s}^{-1}$, using samples with a gauge section of $2.2 \mathrm{~mm}$ and a gauge length of $10 \mathrm{~mm}$. The main data extracted from these curves are given in Tab. 1. This set of curves firstly demonstrated a strong dependence of yield stress on the microstructure, which was the subject of the present work. Important variations in elongation at rupture were also measured. The three specimens AF420, AF 445 and AL073 exhibited an increase of ductility as the yield stress decreased. Astonishingly, the specimen AK784 had very low ductility for an intermediate strength. Such an effect was repeatedly measured in another specimen sintered with the same conditions, to make sure that it did not result from an experimental artifact. The elevated ductility, for a near- $\gamma$ microstructure, achieved with the specimen AL073, has already been obtained a few years ago with a different batch of GE powder having the same chemical composition, thus confirming the exceptional reproducibility and reliability of this sintering process [8].

It is tempting to explain the evolution of yield stress by a variation in the grain size, which can be qualitatively detected on the micrographs of the figure 2 . The grain sizes of all four specimens were measured by TEM following the procedure described in section II. The results given in Tab. 1 confirmed a size effect; i.e., an improvement in the strength of the alloy with a decrease in grain size.

\section{Deformation microstructures}

TEM post-mortem analyses of the deformation microstructure were conducted on the AF 445 specimen deformed up to $1.15 \%$ plastic strain at room temperature. Fig. 3a presents a zone containing $48 \gamma$ grains and $4 \alpha_{2}$ grains (numbered 10,13, 33 and 35), which are delimited in Fig. 3d. When growth twins or ordered domain boundaries divide a zone into several parts, each part is considered as a grain since its orientation is different from its neighboring part. For example, even though they appeared to come from a common former grain, grains 2,3 , and 4 were treated as 3 different grains because some growth-twins divided them, leading to three different orientations. In what follows, the 13 grains having a diameter lower than 1.5 $\mu \mathrm{m}$ (Grains from G25 to G28 and from G37 to G45) will be considered as small grains and studied separately (section IV-2). 
The general view (Fig. 3a) highlights the following points, which will be examined in detail thereafter:

- Two deformation modes involving twins (Fig. 3b) and ordinary dislocations (Fig. 3c ) were activated,

- Numerous interactions between the activated deformation modes and the boundaries were observed,

- Some grains were free of dislocations.

Throughout the paper, the grains will be referenced with the number given in Fig. 3 .

\section{IV-1. Deformation modes}

As illustrated in Fig. 3 b\&c, the two major deformation modes activated during the deformation of this SPS-TiAl alloy at room temperature were the glide of ordinary dislocations and twinning. Ordinary dislocations were elongated along their screw direction and they were anchored at numerous pinning points, as very frequently observed in the $\gamma$ phase of TiAl alloys deformed at room temperature [9-11]. These anchoring points have been ascribed to local chemical heterogeneities [12] and the dislocations' elongation along their screw orientation has been attributed to frictional forces resulting from the dislocation core spreading [11]. The twinning was due to the glide of $a / 6<112]$ Shockley dislocations in (111) plane [13]. Note that $a / 6<211$ ] Shockley dislocations were inoperative as their glide would have produced a complex stacking fault. As a result there was only one operative twinning system by (111) plane. As usual for TiAl alloys deformed at room temperature, superlattice dislocations of a<101] type were scarcely observed. In fact, we observed a few of them in only two grains (G37 \& G47).

The orientation of each of the $48 \gamma$ grains (Fig. 3) was initially determined by conventional diffraction and stereographic analyses. The Schmid's factors of the possible deformation systems were calculated for all the grains based on their orientations. Then, the activated systems were determined using the g.b $=0$ criterion. Concerning ordinary dislocations, one Burgers' vector belongs to two (111) planes. Except in a few cases (for example, when a dislocation pile-up is confined in a same plane), it is long and difficult and not always possible to determine in which plane these dislocations glide. We thus considered that they were gliding in the plane possessing the higher Schmid's factor. On the contrary, as twins are confined in (111) planes, the twinning plane is immediately deduced from the orientation of their traces with respect to that of the grain. As there is only one twinning 
system available per (111) plane, the twinning system is thus fully characterized. Besides, the tension/compression asymmetry implies that a twinning system is compatible with the stress orientation in only one out of two cases. The compatibility of one twinning system with the stress orientation can be verified by conventional TEM using the Final Start Right Hand (FSRH) convention, but this requires a long, meticulous, and difficult procedure impossible to perform repeatedly. Consequently, for the case of twinning, we only discussed the Schmid's factors of the activated systems. Indeed, the non-activated ones were not considered as we did not know whether or not they were due to an incompatibility with the stress orientation.

In the present study, 35 large grains (diameter $>1.5 \mu \mathrm{m}$ ) were analyzed, which corresponded to 70 Burgers vectors of ordinary dislocations available and 70 twinning systems, which should satisfy the tension/compression asymmetry. Forty-six Burgers vectors of ordinary dislocations and 13 twinning systems were found to be activated, indicating a higher facility of movement for the ordinary dislocations. The behavior of grains deforming by ordinary dislocations and twinning will be described in sections IV-3 and IV-4, respectively.

\section{IV-2. Deformation of small grains}

Small grains presented a different deformation microstructure than the larger ones. Among the 13 small grains of the zone, a clear deformation by ordinary dislocations was evidenced in only three of them (G27, G37 \& G38-Fig. 4a). As for larger grains, these ordinary dislocations were screw oriented. Six grains (G25, G26, G28, G39, G40 \&43) were free of deformation (Fig. 4b). Following previous studies [14], these grains, which recrystallized during the SPS process, did not deform during the tensile test at room temperature. In the four other small grains (G41, G42, G44 \& G45), a higher density of dislocations was observed (Fig. 4c), with no clear features such as, for example, an elongation along their screw orientation. Thus, this heavy deformation was attributed to the deformation occurring during SPS densification, which preceded the recrystallization [14 ]. Twins were never observed in these small grains. On the whole, the particular behavior of these small grains has probably to be correlated to the higher strength of microstructures made of small grains, which is measured in the present work. These 13 small grains covered only $18 \%$ of the investigated zone and can probably be bypassed by the deformation. Consequently, they have apparently little influence on the macroscopic behavior of the material as they led to neither stress concentration, nor crack nucleation. In conclusion, small grains had a particular behavior and were not taken in account in the following quantitative analysis. 


\section{IV-3. Ordinary dislocations}

Fig. 5 displays histograms of the Schmid's factors of ordinary dislocation systems either activated (a), or not (b). These histograms were established from the study of the 35 large grains of the zone presented in Fig. 3. It appeared that Schmid's law was generally obeyed as the number of activated systems increased with the Schmid's factor, whereas that of the nonactivated systems decreased. However, some anomalies to Schmid's law were detected.

First, six ordinary dislocation systems were activated although their Schmid's factors were smaller than 0.2. Five of them belonged to grains (G2, G3, G22, G23 \& G24) bordered by at least one growth-twin boundary, with the observed Burgers' vectors lying in the plane of this twin interface. These dislocations were thus able to move from one grain to an adjacent one by crossing the twin boundaries with a classical cross-slip mechanism. Indeed, they were screw oriented and their Burgers' vectors (<110] type) belonged to the plane common to both grains. An example is given in Fig. 6 where three grains, divided by two growth-twin boundaries (TB1 \& TB2), are deformed by rectilinear screw dislocations. Their Burgers' vector were parallel to a close-packed direction of the twin boundary. No dislocation pile-ups were observed in these three grains, but rather a homogeneous distribution, indicating an easy crossing of the boundary by cross-slip. Another situation where the deformation was transmitted from one grain to another one is presented in Fig. 7a. In this case, a twin present in the grain G9 reached a grain boundary causing the emission of an ordinary dislocation loop in the adjacent grain (G4). The nucleation of this loop, part of a system having a Schmid's factor as low as 0.12 , was clearly due to the stress concentration caused by Shockley dislocations pile-up at the grain boundary.

Second, in each of the 7 grains G7, G9, G12, G29, G34, G46, and G49, the ordinary dislocation systems having the highest Schmid's factor (always greater than 0.3) were not activated (Fig. 5b). In five of these grains (G7, G12, G29, G34, \& G49), the second ordinary dislocations system (the one activated), regardless of its Schmid's factor, had its Burger's vector lying in a plane parallel to a growth-twin boundary.

All these results thus demonstrate that the anomalies to Schmid's laws are correlated to an easy nucleation at boundaries and are chiefly observed when the Burgers vectors of the activated dislocations are parallel to the twin growth boundary plane. When the activated dislocation system was not influenced by the deformation of an adjacent grain, Schmid's law was always respected. Fig. 5c\&d present histograms of the Schmid factors for the ordinary 
dislocations of those grains which are not bordered by a twin growth. The limit Schmid factor for the activation of ordinary dislocations is approximately of 0.3 , as previously found in a G4-SPs alloy [14].

\section{IV-4. Twinning}

For twinning, the histogram of the Schmid factors (Fig. 8) indicates that Schmid's law is satisfied. In Fig. 7c, several examples of twin boundary crossing (marked TBC) and grain boundaries crossing (marked GBC) are presented. Three examples of the deformation's continuity through a grain boundary can be observed. In two cases (marked GBC1 and GBC2), an incident twin leads to the emission of a twin in the adjacent grain. In both cases, the emitted twins possess a high Schmid factor $(0.36 \& 0.50)$. Fig. $7 b$ shows one example of two twin boundaries crossed by a twin (G9, G6 \&G7). On both sides, the twins are in planes which are in mirror symmetry with respect to the twin boundary plane. The enlargement (G7) clearly shows a twin tip emitted at the boundary. This situation has been observed several times.

The understanding of these boundary crossing mechanisms is beyond the aim of the present paper. The emission of a twin can be due to either a proper transmission of part of the shearing associated to the incident twin, or to a nucleation of a new twin under the effect of the internal stress resulting from the impingement of the incident twin. This question has been addressed in a previous study for the case of oriented lamellar TiAl alloys [15,16]. In the present study, the perfect alignment of the slip traces in the boundary (exemplified in Fig. 7,b) is seen as an indication of the former process.

For these 13 activated twinning systems, we have identified that they were emitted under the effect on an incident twin at a twin growth boundary in 6 cases and at a general grain boundary in 3 cases. For the 4 other cases, the emission location of the twin was not detected. Interestingly, 9 of the 13 activated twinning systems are in grains bordered by a twin growth boundary.

Fig. 9 shows a twin in the same TEM sample but not in the investigated area. This twin, emitted at a boundary (at the place marked source) as highlighted by the curvature of the dislocations, crossed the whole grain and formed a pile-up at the opposite boundary. In the middle part, the twin overcame an obstacle formed by a dislocation network, which explained the discrepancy in the Shockley dislocation repartition in the twin. These observations illustrate that twins can contain a high number of piled-up Shockley dislocations. 


\section{Discussion}

\section{V-1. Activation of the deformation modes in the rphase}

We showed that the deformation of the $\gamma$ phase in our alloys was due to both the glide of ordinary dislocations and twinning. As specified in section IV-1, in the area presented in the Fig. 3, the number of deformation systems available was 70 in the case of twinning and 70 in the case of ordinary dislocations, i.e. two of each by grain. The study of the different systems actually activated during deformation indicates that the glide of ordinary dislocations is more easily activated than twinning. In the $\gamma$ grains that were not bordered by twin growth boundaries, Schmid's law was correctly satisfied for ordinary dislocations. The minimum Schmid's factor of the ordinary dislocation systems activated was 0.3 , corresponding to a CRSS (Critical Resolved Shear Stress) of $163 \mathrm{MPa}(0.3 \times 544 \mathrm{MPa})$, in the case of the sample AF 445. Remarkably, a similar study in a G4 alloy [14] led to the same minimum Schmid's factor of 0.3, which corresponded in this case to a greater CRSS of $195 \mathrm{MPa}$, probably due to the strengthening effect of heavier alloying elements such as tungsten and rhenium.

The activation of twinning was largely consistent with Schmid's law, with a same minimum Schmid's factor of 0.3. Nevertheless, we observed several examples of the deformation's transmission across grain boundaries by twinning. Similar observations were done by Zghal et al. [15,16] in a lamellar TiAl alloy where the deformation propagated through the lamellae interfaces thanks to twinning, with same features. This analogy suggests that internal stresses, resulting from the pile-up of dislocations at grain boundaries, play an important role in the nucleation of twins. Twinning could also lead to the nucleation of dislocations at grain boundaries even though they belonged to low Schmid's factor dislocation systems (GBC3 in Fig. 7), leading to some non-compliance with the Schmid's law. Moreover, it's worth underlining that, provided that the thickness of the TEM foil $(0.3-0.5 \mu \mathrm{m})$ was much smaller than the average grain size $(1.8 \mu \mathrm{m})$, we actually imaged only a fraction of these particular nucleation events. Likewise, not all the twin nucleation sites were captured. This was especially the case for twins emitted at grain boundaries under the effect of an incident twin. Indeed, the intersection between incident and emitted twins corresponds to a line which is possibly situated outside the thin foil. With this in mind, and extrapolating from our observations, twins, in the bulk sample, were assumed to mostly nucleate at grain boundaries due to internal stresses.

The nature of the grain boundary (either a growth-twin boundary or a boundary between grains without orientation relationship) also played a role in the deformation's transmission 
through the microstructure. First, in the case of ordinary dislocations, the systems activated that did not comply with the Schmid's law were almost all related to either the nucleation of dislocations or to the deformation's transmission through growth-twin boundaries. Second, as illustrated by Fig. 7, the nucleation of a twin at a grain boundary, caused by internal stresses due to another twin in the adjacent grain, was observed at both kinds of grain boundaries but more often at growth-twin boundaries ( 6 of the 9 cases observed). These results indicated that the deformation's propagation through twin boundaries was easier, in consistency with a higher degree of symmetry. They also highlighted that the deformation's transmission across boundaries between grains with no orientation relationship was possible only in the case of twinning, which provided a sufficient number of piled-up dislocations.

The same minimum Schmid's factor of 0,3 was found to be necessary to activate both ordinary dislocations gliding and twinning, although ordinary dislocations were observed more frequently. This showed that twinning required an additional contribution from internal stresses resulting from pile-ups and the fulfilling of geometry conditions on the Burgers vectors and on the glide twinning planes, analyzed in detail for the case of lamellar interfaces $[15,16]$.

\section{V-2. Hall-Petch effect and pile-up model}

The tensile tests performed at room temperature highlighted the influence of the $\gamma$ grain size on the yield stress. In the following interpretation, we ignored the effect of the addition of boron on the plasticity of the $\gamma$ phase as only a very small amount was introduced. Moreover, it appeared to mostly precipitate in the shape of borides, too large and too dispersed to anchor dislocations but sufficient to pin grain boundaries [6]. Likewise, we did not consider the influence of the $\alpha_{2}$ phase, free of deformation after mechanical testing, which represented only a small fraction of the microstructure.

The Hall-Petch theory states that the flow stress $\sigma$ varies with the square root of the average grain size, according to the following relation $\sigma=\sigma_{0}+k / \sqrt{D}$ where $\sigma_{0}$ and $\mathrm{k}$ are constants and $\mathrm{D}$ is the average grain size. $\sigma_{0}$ is assumed to be the stress necessary to move a dislocation in a single-crystal sample and $\mathrm{k}$ is the Hall-Petch constant. Fig. 10 presents a graph of the Yield Stress as a function of the square root of the grain size. This plot leads to $\mathrm{k}=0.98 \mathrm{MPa} \mathrm{m}{ }^{1 / 2}$ and $\sigma_{0}=24 \mathrm{MPa}$. This value of $\mathrm{k}$ is consistent with those measured by Appel et al. [1] (0.91 MPa m $\left.{ }^{1 / 2}\right)$, Huang et al. [17] (1.1 and 1.4 $\left.\mathrm{MPa} \mathrm{m}^{1 / 2}\right)$, Vasudevan et al. [18] (1.37 $\left.\mathrm{MPa} \mathrm{m}{ }^{1 / 2}\right)$, and Bertheau [2] (1.18 $\left.\mathrm{MPa} \mathrm{m}^{1 / 2}\right)$. However, it is higher than those 
measured by Umakoshi et al. [3] (0.50 $\left.\mathrm{MPa} \mathrm{m}^{1 / 2}\right)$ for interfaces of lamellar alloys and by Umakoshi et al. [19] (0.27 $\left.\mathrm{MPa} \mathrm{m}^{1 / 2}\right)$ and Caillard et al. [20] $\left(0.058-0.094 \mathrm{MPa} \mathrm{m}^{1 / 2}\right)$ for ordered domain boundaries. This confirms that grain boundaries are stronger obstacles to the propagation of the deformation than both lamellar interfaces and ordered domains.

The most common explanation for the Hall-Petch effect in polycrystalline metals is the pile-up model. It specifies that the deformation's transmission across multiple grains results from internal stresses caused by pile-ups of $n$ dislocations at the boundaries. Note that this explanation is probably the only one which could explain a high value of the Hall-Petch constant.

The length $L$ of the dislocation pile-up, considered to be half the grain size $\mathrm{D}$, is given by: $L=\frac{n \mu b}{4 \tau}$, where $\mu$ is the shear modulus (65,2 GPa), $b$ the Burgers' vector of the piled-up dislocations, and $\tau$ the resolved shear stress. Both $\tau$ and $\sigma$ are linked by the Taylor's factor as following: $\tau=0,33 \sigma$. This factor of 0,33 is consistent with with the minimum Schmid's factor found, in the present study, to be necessary to activate both ordinary dislocation glide and twinning. The boundary strength verifies: $\sigma_{B}=n \sigma=\frac{2 \sigma^{2} D}{3 \mu b}[20]$.

This leads to $\sigma=\left(\frac{3}{2} \mu b n \sigma\right)^{1 / 2} \frac{1}{\sqrt{D}}$ and thus to $k=\left(\frac{3}{2} \mu b n \sigma\right)^{1 / 2}$.

The number of dislocations contained in the piled-up is therefore $n=\frac{2}{3} \frac{k^{2}}{\mu b \sigma}$.

In the case of the AF 445 specimen $(\sigma=544 \mathrm{MPa})$, these relationships lead to $n=65$ for Shockley dislocations involved in twinning $\left(b=0,16.10^{-9} \mathrm{~m}\right)$, and to $n=37$ for ordinary dislocations $\left(b=0,28.10^{-9} \mathrm{~m}\right)$. These numbers of dislocations correspond to the length of the pile-up of $0.95 \mu \mathrm{m}$ in either cases.

The pile-up model appears to be relevant to explain the Hall-Petch effect in our near- $\gamma$ TiAl alloy, in the case of twinning, due to the following reasons:

i) The calculated length of the dislocation pile-up $(0.95 \mu \mathrm{m})$ is of the order of half the average grain size $(0.9 \mu \mathrm{m})$.

ii) Pile-ups were observed only in the case of twinning as ordinary dislocations were highly subject to cross-slip. Moreover, as illustrated by Fig. 9, twins can contain a large number of dislocations, consistent with our calculations $(n=65)$.

iii) Twins were found to transmit the deformation through grain boundaries in many examples. 
iv) $\sigma_{0}=24 \mathrm{MPa}$ seems to be a reasonable minimum stress necessary to propagate twins in a TiAl single crystal as it is admitted that the controlling process for twinning is the nucleation. For instance, Kawabata et al. [21] found a stress of 75 $\mathrm{MPa}$ for the activation of twinning in a TiAl single crystal.

Even if twins were observed less often than ordinary dislocations, they are necessary to ensure a three-dimensional deformation of the material since the ordinary dislocations' glide only leads to shearing in the (001) basal plane of the quadratic $\mathrm{L}_{0}$ structure. This could explain why twinning controls the transmission of the deformation and leads to the measured size effects, in theoretical consistency with the pile-up model.

\section{Conclusions}

Microstructures, tensile properties at room temperature, and deformation modes of near- $\gamma$ TiAl alloys were studied. Four specimens with average grain sizes ranging from $0.7 \mu \mathrm{m}$ to 3.2 $\mu \mathrm{m}$ were sintered by Spark Plasma Sintering. In one of them, a zone containing $48 \gamma$ grains was analyzed by transmission electron microscopy to determine both size and orientation of these $\gamma$ grains and to characterize the deformation systems activated. The latter appeared to be glide of ordinary dislocations and twinning, which satisfied partially or totally Schmid's law, respectively. Twins were found to propagate the deformation across grain boundaries due to internal stresses resulting from the pile-up of Shockley dislocations. Due to their ability to cross-slip intensively, ordinary dislocations did not form any pile-ups. These results will be used to model the mechanical properties of these TiAl alloys with micromechanical modeling and the finite-element method.

A size effect, namely the influence of grain size on the strength of the material, was measured. The yield stress at room temperature increased strongly as the grain size decreased, leading to a high Hall-Petch constant of $0.98 \mathrm{MPa} \mathrm{m}^{1 / 2}$. These results were interpreted by the pile-up model applied to Shockley dislocations involved in twinning, based on a good match between calculations and experiments. Twinning, required to insure a three-dimensional deformation, appeared to be the process controlling the mechanical behavior of our near- $\gamma$ TiAl alloys. 


\section{References}

[1] Appel F., Paul J., Oehring M., Gamma Titanium Aluminides: Science and Technology, (C) Wiley-VCH Verla GmbH \&Co. KGaA, 2011.

[2] Bertheau O., Etude des mécanismes d'écrouissage et d'endommagement cycliques des alliages TiAl élaborés par métallurgie des poudres, Thèse de l'Université de Poitiers, 25 Octobre 2005.

[3] Umakoshi Y., Nakano T., Yamane T., The effect of orientation and lamellar structure on the plastic behaviour of TiAl crystals, Materials Science and Engineering A, $152,(1992), 81-88$.

[4] Parthasarathy T.A., Keller M., Mendiratta M.G., The effect of lamellar lath spacing on the creep behavior of Ti-47at\% Al , Scripta Met, 38 (1998) 1025-1031.

[5] Voisin T, Durand L, Karnatak N, Le Gallet S, Thomas M, Le Berre Y, Castagne JF, Couret A, Temperature control during Spark Plasma Sintering and application to up-scaling and complex shaping, Journal of Materials Processing Technology, 213 (2013) 269-278.

[6] Luo J.S., Voisin T., Monchoux JP., Couret A., Refinement of lamellar microstructures by boron incorporation in GE-TiAl alloys processed by Spark Plasma Sintering, Intermetallics, 36 (2013) 12-20.

[7] Zghal S., Naka S., Couret A., A quantitative tem analysis of the lamellar microstructure in TiAl based alloys, Acta metall. mater., 45 (1997) 3005-3015.

[8] Couret A., Molénat G., Galy J., Thomas M., 2008, Microstructures and mechanical properties of TiAl alloys consolidated by spark plasma sintering, Intermetallics, 16, (2008) 1134-1141.

[9] Viguier B., Hemker K.J., Bonneville J., Louchet F., Martin J.L., Modeling the flow stress anomaly in gamma-TiAl 1.Experimental observations of dislocations mechanisms, Phil. Mag. A, 71 (1995) 1295-1312.

[10] Sriram S., Dimiduk D.M., Hazzledine P.M., Vasudevan V.K., The geometry and nature of pinning points of 1/2 (110] unit dislocations in binary TiAl alloys Phil. Mag. A, 76 (1997) 965-993

[11] Couret A., An in-situ study of ordinary dislocation glide in gamma-TiAl alloys,_Phil. Mag. A, 79 (1999) 1977-1994.

[12] Zghal S., Menand A., Couret A., Pinning points anchoring ordinary and Shockley dislocations in TiAl alloys, Acta Mat., 46 (1998) 5899-5905.

[13] Farenc S., Coujou A., Couret A., An in situ study of twin propagation in TiAl, Phil. Mag. A, 67 (1993) 127-142.

[14] Jabbar H., Monchoux JP., Thomas M., Couret A., Microstructures and deformation mechanisms of a G4 TiAl alloy produced by spark plasma sintering, Acta Materialia, 59 (2011) 7574-7585.

[15] Zghal S., Coujou A., Couret A., Transmission of the deformation through gammagamma interfaces in a polysynthetically twinned TiAl alloy - I. Ordered domain interfaces (120 degrees rotational), Phil. Mag. A, 81 (2001) 345-364.

[16] Zghal S., Couret A, Transmission of the deformation through gamma-gamma interfaces in a polysynthetically twinned TiAl alloy - II. Twin interfaces (180 degrees rotational), Phil. Mag. A, 81 (2001) 365-382.

[17] Huang SC, Hall EL, The effects of $\mathrm{Cr}$ additions to binary TiAl-Base alloys, Metallurgical Transaction A, 22 (1991) 2619-2667. 
[18] Vasudevan V.K, Court A, Kurath P, Fraser H.L., Effect of grain-size and temperature on the yield stress of the intermetallic compound TiAl, Scripta Metallurgica, 23 (1989) 467-469.

[19] Umakoshi Y, Nakano T, The role of ordered domains an slip mode of alpha-2 phase in the plastic behavior of TiAl crystals containing oriented lamellae, Acta metall. mater., 41 (1993) 1155-1161.

[20] Caillard D, Couret A, The Hall-Petch Law Investigated by Means of In Situ Straining Experiments in Lamellar TiAl and Deformed Al, Microscopy Research and Technique, 72 (2009) 261-269.

[21] Kawabata T, Kanai T, Ozumi O, Positive temperature-dependence of the yield stress in TiAl $\mathrm{L}_{0}$ type superlattice intermetallic compound single crystals at 293-1273K, Acta metall. mater., 33 (1985) 1355-1366. 


\section{Figure caption.}

Fig. 1: Comparison of results obtained by EBSD-SEM and TEM. (a) general view by TEM; (b) drawing of the grains, (c) EBSD analysis, (d) ordered domain related grains, (e) growth-twin, and (f) small grains.

Fig. 2: SEM micrographs of the four samples with a near- $\gamma$ microstructure (a) to (d), and corresponding room-temperature tensile properties (e).

Fig. 3: Area where the deformation microstructure was studied. (a) general view by TEM; (b) enlargement showing some twins; (c) enlargement showing some ordinary dislocations, and (d) drawing of the grains.

Fig. 4: Small grains. (a) a grain deformed by ordinary dislocations, (b) a recrystallized grain, and (c) a grain deformed during the SPS densification.

Fig. 5: Histogram of Schmid's factors for ordinary dislocations. (a) and (b) histograms of the activated systems and non-activated ones for all of the 35 grains, (c) and (d) histograms of the activated systems and non-activated ones for grains which were not bordered by a growth-twin boundary.

Fig. 6: TEM micrograph of an area in which ordinary dislocations were able to cross growthtwin boundaries by cross-slip as their Burgers' vector was contained in a plane parallel to the twin interface.

Fig. 7: Examples of the deformation's transmission through grain boundaries. (a) an incident twin in G9 led to the emission of an ordinary dislocation loop in G4, (b) a twin that crossed the G9/G6 and G6/G7 boundaries. The enlargement shows the twin tip, indicating the propagating direction, and (c) several examples of boundaries crossing.

Fig. 8: Histogram of Schmid's factors for twinning.

Fig. 9: A twin containing a lot of Shockley dislocations in a grain of the AF445 specimen, which did not belong to the wider zone investigated shown in Figure 3.

Fig. 10: Hall-Petch plot. 
Table 1: Mechanical properties at room temperature and grains sizes of the four specimens investigated in the present study. $\mathrm{T}_{\mathrm{s}}$ : temperature of the sample during the plateau of the SPS cycle, GS: grain size, YS: Yield stress, UTS: Ultimate stress and A: elongation at rupture.

\begin{tabular}{|l|c|c|c|c|c|}
\hline & $\mathrm{T}_{\mathrm{s}}\left({ }^{\circ} \mathrm{C}\right)$ & $\mathrm{GS}(\mu \mathrm{m})$ & YS $(\mathrm{MPa})$ & UTS $(\mathrm{MPa})$ & $\mathrm{A}(\%)$ \\
\hline AF420 & 1200 & 0.7 & 930 & 946 & 0.45 \\
\hline AK784 & 1274 & 1.0 & 750 & 776 & 0.12 \\
\hline AF445 & 1217 & 1.8 & 544 & 578 & 1.15 \\
\hline AL073 & 1312 & 3.2 & 482 & 568 & 2 \\
\hline
\end{tabular}

